\title{
Surrogate model uncertainty in wind turbine reliability assessment
}

\section{Journal Article}

\section{Author(s):}

Slot, René M.M.; Sørensen, John D.; Sudret, Bruno (D); Svenningsen, Lasse; Thøgersen, Morten L.

Publication date:

2020-05

Permanent link:

https://doi.org/10.3929/ethz-b-000394728

\section{Rights / license:}

Creative Commons Attribution-NonCommercial-NoDerivatives 4.0 International

\section{Originally published in:}

Renewable Energy 151, https://doi.org/10.1016/j.renene.2019.11.101 


\section{GHzürich}

\section{SURROGATE MODEL UNCERTAINTY IN WIND TURBINE RELIABILITY ASSESSMENT}

R.M.M. Slot, J. D. Sørensen, B. Sudret, L. Svenningsen, M.L. Thøgersen

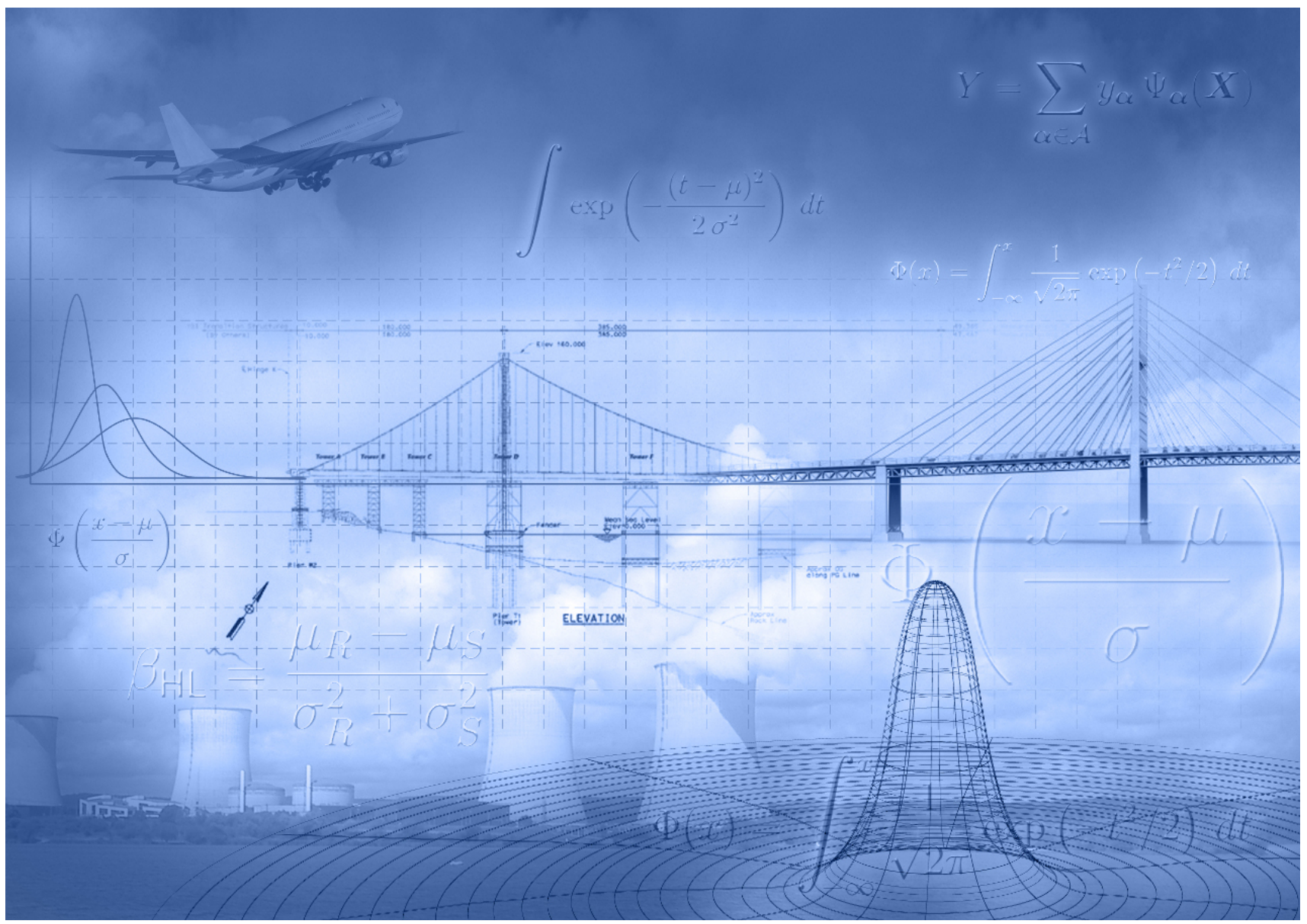




\section{Data Sheet}

Journal: $\quad$ Renewable Energy

Report Ref.: RSUQ-2019-005

Arxiv Ref.:

DOI: $\quad$ 10.1016/j.renene.2019.11.101

Date submitted: June 25, 2019

Date accepted: $\quad$ November 18, 2019 


\title{
Surrogate Model Uncertainty in Wind Turbine Reliability Assessment
}

\author{
René M. M. Slot ${ }^{1,3}$, John D. Sørensen ${ }^{1}$, Bruno Sudret ${ }^{2}$, Lasse Svenningsen ${ }^{3}$, and Morten L. \\ Thøgersen ${ }^{3}$ \\ ${ }^{1}$ Department of Civil Engineering, University of Aalborg, Aalborg, 9220, Denmark \\ ${ }^{2}$ Chair of Risk, Safety and Uncertainty quantification, ETH, Zürich, Switzerland \\ ${ }^{3}$ EMD International A/S, Aalborg, 9220, Denmark
}

Correspondence to: René M. M. Slot (rmms@ civil.aau.dk)

\begin{abstract}
Lowering the cost of wind energy entails the optimization of wind turbine material consumption without compromising structural safety. Traditionally, wind turbines are designed by the partial safety factor method, which is calibrated by probabilistic models and presented in the IEC 61400-1 design standard. This approach significantly reduces the amount of aeroelastic simulations required to assess the fatigue limit state of wind turbines, but it may lead to inconsistent reliability levels across wind farm projects. To avoid this, wind turbines may be designed by probabilistic methods using surrogate models to approximate fatigue load effects. In this approach, it is important to quantify and model all relevant uncertainties including that of the surrogate model itself. Here we quantify this uncertainty according to Eurocode 1990 for polynomial chaos expansion (PCE) and Kriging using wind data from 99 real sites and the $5 \mathrm{MW}$ reference turbine designed by NREL. We investigate a wide range of simulation efforts to train the surrogate models. Our results show that Kriging yields a higher accuracy per invested simulation compared to PCE. This improved understanding of utilizing PCE and Kriging in fatigue reliability assessment may significantly benefit decision support in probabilistic design of wind turbines.
\end{abstract}

Key words: Wind turbine, Fatigue loads, Structural reliability, Surrogate models, Model uncertainty

\section{Introduction}

To lower the cost of wind energy it is important to utilize wind turbines to their full load bearing capacity, but without compromising structural safety. A typical design approach is to use the partial safety factor method, calibrated by fully probabilistic models and presented in standardized codes as the IEC 61400-1 design standard for wind turbines [1]. This semiprobabilistic approach accounts for variability and uncertainty in strength and load parameters via characteristic values defined by quantiles. A final design equation is then adjusted by partial safety factors in order to meet a target structural reliability level, which is defined with consideration of economic loss and risk of human lives to optimize material consumption from a societal point of view [2]. This simplified framework provides a direct advantage in computational requirements to assess whether a given wind turbine class is suited for a particular site and park layout. However, it may lead to inconsistent reliability levels as the simple characteristic input cannot fully explain the variation of the load response across all load 
bearing components [3,4]. Partial safety factors are, therefore, typically calibrated based on conservative assumptions. As a result, it can be expected that wind turbines on average are over-designed, thereby leading to a higher cost of wind energy than necessary. To avoid this excessive use of materials, site-specific assessment of wind turbines can be based directly on probabilistic methods as described in the recent $4^{\text {th }}$ edition of the IEC $61400-1$ design standard [1].

The main challenge in probabilistic design of wind turbines compared to the traditional approach is that significantly more load evaluations are required. In particular, fatigue analysis during normal operation (design load case 1.2 [1]) involves an unfeasibly large amount of load simulations as fatigue damage accumulates during the entire lifetime of the turbine. It is therefore necessary to assess the integrated fatigue load across the full joint wind climate distribution. For onshore wind turbines this includes at least: wind direction $(\theta)$, wind speed $(U)$, turbulence $\left(\sigma_{U}\right)$, vertical wind shear $(\alpha)$, air density $(\rho)$ and flow inclination $(\varphi)$ [5]. Consequently, the sheer amount of aero-elastic simulations required to fully evaluate the lifetime fatigue load imposes a computational barrier to probabilistic design [6]. To circumvent this barrier a shortcut from wind climate to wind turbine fatigue loads is needed. Various methods have been proposed to simplify wind turbine fatigue load assessment via surrogate models, also referred to as meta-models, response surfaces or proxies. This motivated Dimitrov et al. [7] to benchmark the accuracy of several surrogate techniques, with emphasis towards prediction of lifetime fatigue loads. Their study included importance sampling, quadratic regression, nearest-neighbour interpolation, polynomial chaos expansion (PCE) and Kriging. In conclusion, Kriging and PCE were superior with Kriging being the most accurate model, but its computational time also exceeds that of PCE when used as a predictor.

Surrogate models make fully probabilistic design of wind turbines viable. Toft et al. [8] used a quadratic response surface for reliability analysis of onshore wind turbines to model the uncertainties in wind climate assessment, aleatory as well as epistemic, and quantified their importance. Morató et al. [9] established a Kriging model to capture Von Mises stresses and thereby assess the structural reliability of offshore wind turbines in the ultimate limit state. In addition, they investigated the influence of the computational effort (number of samples and seeds) used to calibrate the surrogate model. With focus on offshore wind turbine fatigue loads Teixeira et al. [10] used a Kriging model to analyse the importance of different wind and wave climate parameters. Murcia et al. [11] used the uncertainty propagation properties of PCE to analyse the sensitivity of the wind climate on the power output and structural response of an onshore turbine. Focussing on blade design, Hu et al. [12] proposed a reliability-based design optimization which relied on multiple Kriging models to predict fatigue loads at critical structural hotspots. They included wind climate uncertainty, spatial as well as temporal, while also considering manufacturing uncertainties of the composite laminate.

A common goal of existing literature on wind turbine fatigue reliability is to establish novel reliability models and quantify the long chain of uncertainties from wind climate assessment to wind turbine load effects. In this context, an important uncertainty is still missing in the literature, namely the use of a surrogate model to approximate fatigue loads instead of direct aero-elastic simulations. The scope of this paper is to study this uncertainty by using wind measurements from 99 real wind turbine sites, which constitutes a solid base to quantify a general uncertainty model for future applications. In this work PCE and Kriging are considered 
due to their very promising capabilities in terms of capturing fatigue loads, propagating uncertainty and carrying out sensitivity analyses, whilst minimising computational requirements. The chosen techniques also represent two main approaches to predict a model output viz. regression (PCE) and interpolation (Kriging).

\section{Wind Measurements}

High quality 10 min. measurements of wind direction, windspeed, turbulence and wind shear from 99 real wind turbine sites are used in this study. All measurement campaigns are from meteorological masts or from wind power projects and reflect typical setups used for load calculations in practice. Collectively, the masts represent a wide geographical spread with varying terrain complexity and roughness fetches. The measurements were also used by Slot et al. [3] where a detailed description of the data can be found.

\subsection{Ambient joint wind climate}

The site-specific joint distributions of wind direction, windspeed, turbulence and wind shear are described in terms of conditional distributions as summarized in Table 1. Measurements leading to air density were unavailable at most sites, so for consistency air density time series are estimated from a meso-scale model. Flow inclination measurements were also missing, and for simplicity these are modelled as a fixed value dependent on the site-specific orography, see Table 2. It is noted that air density as well as flow inclination have very limited influence on fatigue loads when compared to wind speed, turbulence and wind shear $[3,4,13]$, hence, the applied simplifications are not expected to significantly affect the results in this paper.

Table 1: Joint wind climate distribution

\begin{tabular}{|l|c|l|}
\hline Wind climate parameter & Notation & Description \\
\hline Wind direction & $P_{\theta}(\theta)$ & Discrete distribution [5] \\
\hline Wind speed & $f_{U}(U \mid \theta)$ & Weibull distribution [5,14] \\
\hline Turbulence & $f_{\sigma_{U}}\left(\sigma_{U} \mid U, \theta\right)$ & Lognormal distribution [5] \\
\hline Wind shear & $f_{\alpha}\left(\alpha \mid \sigma_{U}, U, \theta\right)$ & Normal distribution [4,13] \\
\hline Air density & $f_{\rho}(\rho)$ & Normal distribution [4] \\
\hline Flow inclination & $\varphi$ & Fixed value \\
\hline
\end{tabular}

The conditional distributions of wind speed are derived by binning the wind direction into twelve sectors covering $30^{\circ}$ each as recommended in the IEC 61400-1 standard [5], and the

\footnotetext{
${ }^{a}$ A Weibull distribution may also be considered as recommended in the IEC 61400-1 ed. 4 design standard.
} 
conditional distributions of turbulence are determined by further binning wind speed by $1 \mathrm{~m} / \mathrm{s}$. To model wind shear dependent on direction, wind speed, and turbulence the procedure described by Dimitrov et al. [13] is adopted. In each wind speed and directional bin, the available turbulence samples are ranked and divided into five equally sized intervals, each assumed to represent a width of $20 \%$ of the turbulence cumulative distribution function. Then, in each of the turbulence intervals a normal distribution is fitted to the available wind shear data.

The first and second moment of the turbulence and wind shear data are required in each defined bin to estimate the conditional distributions. To ensure robust estimates of the moments only direction and wind speed bins with 50 or more samples are considered. In bins with less than 50 samples the distribution parameters are extrapolated in order to have a complete description of the joint wind climate across the entire range of normal turbine operation. Following the IEC 61400-1 standard [1], the mean value $\left(\mu_{\sigma_{U}}\right)$ and standard deviation $\left(\sigma_{\sigma_{U}}\right)$ of the turbulence distributions are extrapolated by using linear models. This is outlined by Eqs. (1) and (2), where $a$ and $b$ are calibrated to obtain the best least square fit to the data in accepted bins.

$$
\begin{aligned}
& \mu_{\sigma_{U}}(U \mid \theta)=a_{\mu}(\theta) \cdot U+b_{\mu}(\theta) \\
& \sigma_{\sigma_{U}}(U \mid \theta)=a_{\sigma}(\theta) \cdot U+b_{\sigma}(\theta)
\end{aligned}
$$

To extrapolate the mean value of the wind shear distribution $\left(\mu_{\alpha}\right)$ it is approximated by the median of $\mu_{\alpha}$ over the three highest accepted wind speed bins given direction and turbulence. This imitates how atmospheric stability turns towards neutral conditions at medium to high wind speeds where the mean wind shear becomes almost constant [15]. Finally, the standard deviation of the wind shear distribution is extrapolated. This is inversely proportional to wind speed as shown in Eq. (3), where $c$ is calibrated by the available data [16].

$$
\sigma_{\alpha}\left(U \mid \theta, \sigma_{U}\right)=\frac{c_{\alpha}\left(\theta, \sigma_{U}\right)}{U}
$$

Table 2: Flow inclination model

\begin{tabular}{|l|l|l|l|}
\hline Site-specific orography & Flat terrain & Hilly terrain & Steep terrain \\
\hline Fixed flow inclination & $0^{\circ}$ & $6^{\circ}$ & $12^{\circ}$ \\
\hline Number of sites & 62 & 27 & 10 \\
\hline
\end{tabular}

\subsection{Wake added turbulence}

All the included measurements represent ambient climates. This does not mirror the reality of most turbines, where wakes are present in certain directions. Wake added turbulence is, therefore, considered by assuming a rectangular grid layout where a neighbouring turbine is placed 5 rotor diameters $(R D)$ up- and downwind in the main wind direction, and $3 R D$ 
perpendicular to that. The wake added turbulence $\left(\sigma_{U, \text { wake }}\right)$ is modelled according to the IEC 61400-1 standard [1] as outlined in Eq. (4), where $C_{T}$ is the thrust coefficient.

$$
\sigma_{U, \text { wake }}(U, \theta)=\frac{U}{1.5+\frac{0.8 R D(\theta)}{\sqrt{C_{T}(U)}}}
$$

To significantly reduce the computations required in this paper, but without loss of generality, the wakes are assumed to perfectly align with the defined sectors regardless of the distance between the two turbines. This is a simplification, but the main purpose of introducing the wakes is to reproduce a real case representative range of turbulence values. The ambient and wake added turbulence are combined to create the total turbulence that is experienced by the turbine $\left(\sigma_{U, T}\right)$ by Eq. (5).

$$
\sigma_{U, T}(U, \theta)=\sqrt{\sigma_{U}(U, \theta)^{2}+\sigma_{U, w a k e}(U, \theta)^{2}}
$$

\section{Probabilistic Model for Fatigue Failure}

The key objective in probabilistic design is to assess the structural reliability of a given failure mode and check if it meets the target reliability level. This requires a representative limit state equation (LSE) to quantify the failure probability by modelling relevant uncertainties on strengths and loads. In this section a simple yet representative LSE for wind turbine fatigue failure is presented.

\subsection{Wind turbine simulation and fatigue loads}

First, it is relevant to discuss how fatigue loads are calculated. In this paper the framework of "damage equivalent loads" $(D E L)$ is adopted, which implicitly assumes that fatigue strength of materials can be modelled by a linear $S N$-curve, and that Miner's rule [17] may be used to accumulate fatigue damage from varying load effect amplitudes. All fatigue loads are based on 10 min. effective ${ }^{\mathrm{b}}$ simulations of the 5MW reference wind turbine designed by NREL [18]. The turbine's baseline controller is considered, which represents a simple version of typical commercial solutions by using optimal torque control between cut-in and rated wind speed, and collective pitch control between rated and cut-out wind speed $[18,19]$. The turbine is simulated in the aero-servo-elastic software FAST [20], and each realized wind field is computed in TurbSIM [21] using the Kaimal spectrum [22]. The output of the simulations are time-series of load effects for various sensors on the main components of the turbine, which are reduced to a spectrum of load effect amplitudes $\left(\Delta F_{i}\right)$ and a corresponding number of cycles $\left(n_{i}\right)$ by Rainflow counting [23]. This is then further condensed to a single scalar, the $D E L$, which represents the load effect range that produces the same fatigue damage as the entire spectrum. The DEL is outlined in Eq. (6) where the wind climate parameters, except turbulence, are

\footnotetext{
b The term "effective" is used to indicate that transient start-up behaviour is removed from the output.
} 
gathered in the vector $\bar{C}=[U, \alpha, \rho, \varphi]$ to ease notation. Note that the equivalent number of cycles, $N_{e q}$, is used as a reference value and may be selected arbitrarily if it is kept the same when comparing different $D E L$ s.

$$
\operatorname{DEL}\left(\bar{C}, \sigma_{U, T}\right)=\left(\frac{1}{N_{e q}} \sum_{i} n_{i} \Delta F_{i}^{m}\right)^{1 / m}
$$

The combined site-specific equivalent fatigue load $\left(F_{\text {eq }}\right)$ with a one year reference period $\left(T_{\text {ref }}\right)$ is assessed by Eq. (7), where $f_{\text {Site }}$ models the joint wind climate distribution and $T_{\text {sim }}$ is the simulation time.

$$
F_{\text {eq, direct }}=\left(\frac{T_{r e f}}{T_{\text {sim }}} \sum_{\theta} P_{\theta}(\theta) \int_{\sigma_{U}} \int_{\bar{C} \in \mathbb{R}^{4}} f_{\text {Site }}\left(\bar{C}, \sigma_{U} \mid \theta\right) D E L\left(\bar{C}, \sigma_{U, T}\right)^{m} d \bar{C} d \sigma_{U}\right)^{1 / m}
$$

To limit the amount of results shown in this paper only the six main sensors listed in Table 3 are considered. The sensors represent varying sensitivities to the wind climate parameters and controller actions, and collectively they reflect the overall path of the wind loads from acting on the blades until being reacted by the foundation. The fatigue strength of each component is modelled by typical Wöhler exponents used throughout the literature $[4,11,13,24]$.

Table 3: Wind turbine sensors

\begin{tabular}{|l|l|l|l|l|}
\hline Component & Sensor description & Notation & Unit & $\begin{array}{l}\text { Wöhler } \\
\text { exponent }\end{array}$ \\
\hline Blade & Blade root flap-wise bending & RootMyb1 & $\mathrm{kNm}$ & 10 \\
\hline Blade & Blade root edge-wise bending & RootMxb1 & $\mathrm{kNm}$ & 10 \\
\hline Main shaft & Low speed shaft torque & LSSGagMxa & $\mathrm{kNm}$ & 6 \\
\hline Tower (top) & Yaw bearing tilt & YawBrMyp & $\mathrm{kNm}$ & 4 \\
\hline Tower (top) & Yaw bearing yaw & YawBrMzp & $\mathrm{kNm}$ & 4 \\
\hline Tower (bottom) & Tower bottom fore-aft bending & TwrBsMyt & $\mathrm{kNm}$ & 4 \\
\hline
\end{tabular}

\subsection{Limit state equation}

By assessing fatigue loads via direct aero-elastic simulation a LSE for fatigue failure $\left(g_{\text {direct }}\right)$ is defined as shown in Eq. (8), where the design parameter, $z$, relates load effects to stresses and $t$ is time in years [24,25]. 


$$
g_{\text {direct }}(z, t)=\boldsymbol{\Delta}-\frac{N_{\text {eq }} t}{\boldsymbol{K}}\left(\boldsymbol{X}_{\text {Load }} \boldsymbol{X}_{S C F} \frac{F_{\text {eq, direct }}}{Z}\right)^{m}
$$

Bold font indicates if a variable is stochastic. This includes the model uncertainties $\boldsymbol{\Delta}$ and $\boldsymbol{K}$ to account for Miner's rule and the $S N$-approach, respectively, and $\boldsymbol{X}_{L o a d}$ and $\boldsymbol{X}_{S C F}$ to model the uncertainty on wind load effects and stress concentration factors ${ }^{c}$. Typical examples of the uncertainties are presented in Table $4[8,24,25]$.

Table 4. Stochastic models

\begin{tabular}{|l|l|l|c|c|c|}
\hline Variable & Distribution & \multirow{2}{*}{ Expected value } & \multicolumn{3}{|c|}{ Standard deviation } \\
\cline { 4 - 7 } & & & $\mathbf{m}=\mathbf{4}$ & $\mathbf{m}=\mathbf{6}$ & $\mathbf{m}=\mathbf{1 0}$ \\
\hline $\boldsymbol{\Delta}$ & Normal & 1 & 0.30 & 0.40 & 0.50 \\
\hline $\boldsymbol{X}_{\text {Load }}$ & Lognormal & 1 & 0.15 & 0.15 & 0.15 \\
\hline $\boldsymbol{X}_{S C F}$ & Lognormal & 1 & 0.10 & 0.15 & 0.15 \\
\hline $\log \boldsymbol{K}$ & Normal & - & 0.20 & 0.15 & 0.25 \\
\hline
\end{tabular}

The accumulated failure probability $\left(P_{f}\right)$ and associated reliability index $(\beta)$ of the considered component is estimated by Eq. (9), where $\Phi$ models the cumulative standard normal distribution.

$$
P_{f}=\Phi(-\beta)=P(g(z, t) \leq 0)
$$

To evaluate the failure probability, the integrated fatigue load across the entire joint wind climate has to be estimated. Direct aero-elastic simulation for this application is extremely computationally demanding, and in nearly all cases practically unfeasible. This barrier may be overcome by the use of surrogate models as discussed in the introduction. To reiterate, surrogate models aim to accelerate the evaluation of site-specific fatigue loads $\left(F_{\text {eq,proxy }}\right)$, but with a reduced accuracy compared to direct simulation. This should be properly accounted for in the LSE by introducing an additional model uncertainty related to the surrogate model itself $\left(\boldsymbol{X}_{\text {proxy }}\right)$. The uncertainty is related directly to the load effect, similar to $\boldsymbol{X}_{\text {Load }}$ and $\boldsymbol{X}_{S C F}$, and is applied alongside these as shown in Eq. (10).

$$
g_{\text {proxy }}(z, t)=\Delta-\frac{N_{e q} t}{\boldsymbol{K}}\left(\boldsymbol{X}_{\text {Load }} \boldsymbol{X}_{S C F} \boldsymbol{X}_{\text {proxy }} \frac{F_{\text {eq,proxy }}}{z}\right)^{m}
$$

\section{Surrogate Models for Fatigue Load Prediction}

The surrogate model uncertainty depends on the surrogate model type and how many simulations that are invested to train it [7]. Two surrogate techniques are included in this work, specifically Kriging and PCE, and both are implemented in the general purpose uncertainty quantification framework UQLab [26]. This section outlines the input domain and the

\footnotetext{
${ }^{\mathrm{c}}$ The linear relationship between load effects and stresses is based on simple beam theory. To account for nonlinear effects a "stress concentration factor" is typically applied.
} 
experimental design used to train the models, followed by a brief summary of each surrogate technique with emphasis on their configuration in this particular work. For a more general explanation of the details and theory behind Kriging we refer to Santner et al. [27] and for PCE we refer to Sudret [28].

\subsection{Input domain and experimental design}

Regardless of the surrogate model technique it is necessary to sample an experimental design. On the one hand, this requires an input domain that covers the joint wind climate distributions across all 99 sites to avoid extrapolation by the surrogate models which may lead to erratic results. On the other hand, the input domain should also encompass physically realistic wind climate combinations to ensure validity of the aero-elastic simulations used to estimate the output fatigue loads. An input domain that meets these two key objectives was defined by Dimitrov et al. [7], partly based on theoretical considerations of atmospheric stability. In this work, we have chosen to tailor the input domain explicitly to the 99 available sites by using all data including wakes. This is illustrated in Figure 1 for turbulence intensity ${ }^{\text {d }}$ (i.e. $10 \mathrm{~min}$. wind speed coefficient of variation) and wind shear as function of wind speed. The bounds (blue lines) are based on approximations to the extreme quantiles of all data (red lines) with a slight conservative offset.
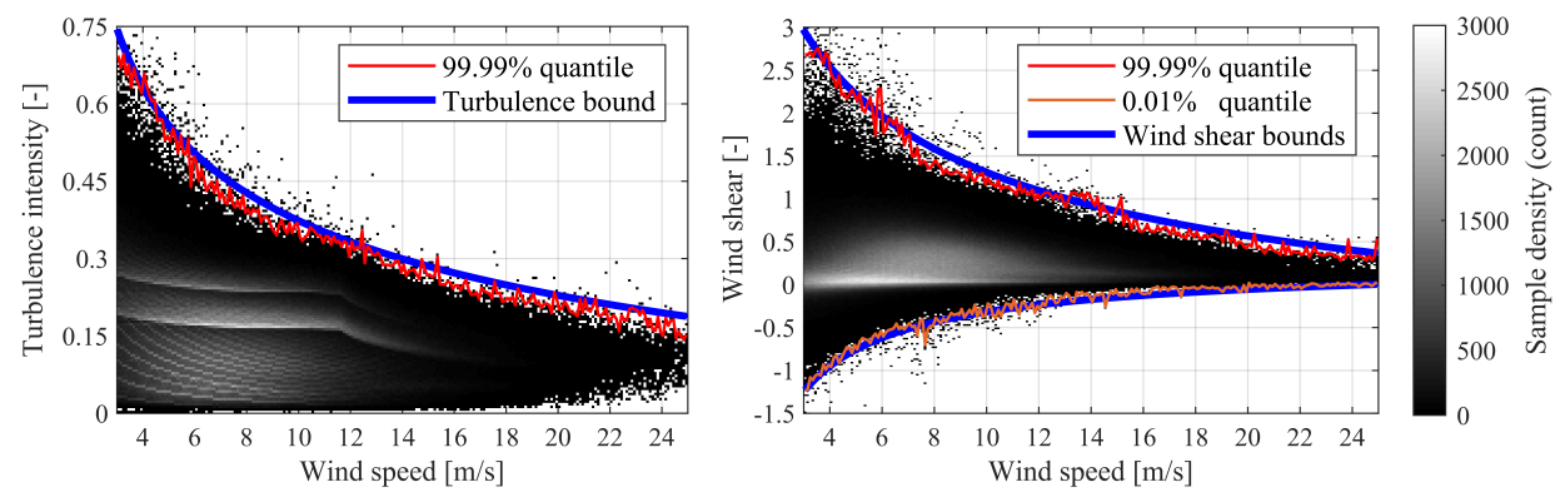

Figure 1: Turbulence bound (left) and wind shear bounds (right) as function of wind speed based on all available measurements. The pronounced clear lines inside the turbulence samples at 0.2 and 0.3 correspond to the wake added turbulence at $3 \mathrm{RD}$ and $5 \mathrm{RD}$. The smaller clear lines are a product of the decimal truncation when the wind measurements are logged.

The bounds on air density are based on the meso-scale modelled data as shown in Figure 2, and the bounds on flow inclination are based on engineering judgement in the interval from $-16^{\circ}$ to $16^{\circ}$.

\footnotetext{
$\mathrm{d}$ Turbulence intensity is used such that the slight offset of the bound accounts for the increasing scale of turbulence with increasing wind speed.
} 


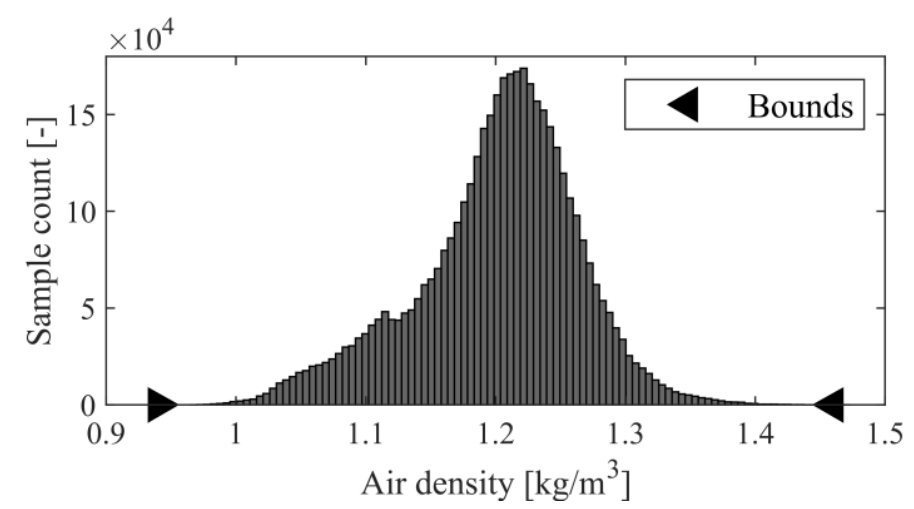

Figure 2: Air density bounds based on all available measurements.

Together, the defined bounds enclose a hyper-volume in the 5-dimensional space of the considered wind climate parameters. An experimental design consisting of 625 samples is drawn uniformly inside this hyper-volume using a quasi-random Halton sequence. This ensures a good space-filling for the entire experimental design, and also when only a subset of the experimental design is utilized. To avoid clear patterns in the Halton sequence, which may compromise the accuracy of the surrogate models, a reverse-radix scrambling is performed as described by Kocis and Whiten [29]. The experimental design is shown in Figure 3. Each of the corresponding DELs is estimated using 100 seeds, resulting in a total of 62,500 aero-elastic simulations.

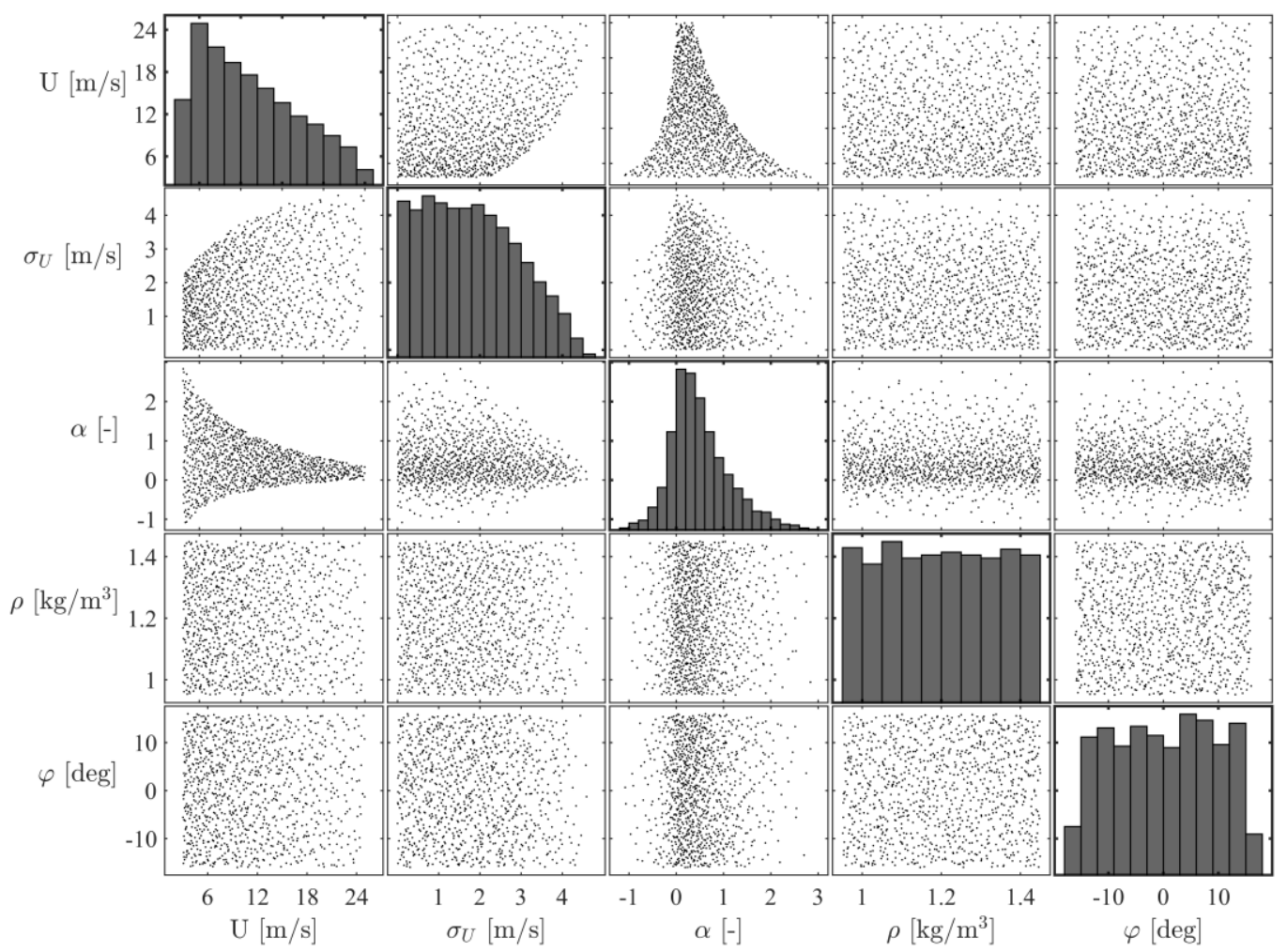

Figure 3: Experimental design with 625 samples drawn uniformly inside the bounded hyper-volume of the wind climate parameters. 


\subsection{Polynomial chaos expansion}

In this work the Wiener-Askey generalized PCE is considered [30]. Generally, it may be used to approximate a function $(\mathrm{Y})$ of a random vector $(\overline{\boldsymbol{X}})$ in dimension $D\left(\overline{\boldsymbol{X}} \in \mathbb{R}^{D}\right)$ by an infinite expansion of a multivariate orthonormal polynomial basis $(\bar{\Psi})$ with respect to the joint probability density function of the input. This is outlined in Eq. (11) where $j$ is a multi-index of the components of the multivariate polynomials.

$$
\mathrm{Y}(\overline{\boldsymbol{X}})=\sum_{j \in \mathbb{N}^{D}} c_{j} \Psi_{j}(\overline{\boldsymbol{X}})
$$

The polynomial basis is built from a set (family) of univariate orthonormal polynomials with respect to each input variable, and classical families have been developed which cover common input distributions [30]. For simplicity, the experimental design is transformed into the standard uniform space by Rosenblatt transformation [31]. Accordingly, only the Legendre family of orthonormal polynomials is required to build the PCE.

For practical application, the infinite sum of polynomials in Eq. (11) is truncated using a hybrid least angle regression algorithm to penalize higher order terms combined with a hyperbolic truncation scheme to disregard insignificant interactive terms, details of which are shown in Blatman and Sudret [32]. By considering polynomial degrees up to 20, the PCE which minimizes the "leave one out" cross-validation error $\left(\epsilon_{L O O}\right)$ is selected following the implementation in UQLab [33]. Note that the $\epsilon_{L O O}$ is chosen as the optimization metric to increase robustness towards over-fitting of the PCE when high-order polynomials are considered.

\subsection{Kriging}

Kriging, also refered to as Gaussian process regression, is a stochastic interpolation technique which assumes the model output $(Y)$ to be a realization of a deterministic mean defined by a regression model $\left(\bar{\beta}_{K R G} \bar{f}_{K R G}^{T}\right)$ and a correlated stochastic process (Z), see Eq. (12) [27].

$$
Y(\bar{X})=\bar{\beta}_{K R G} \bar{f}_{K R G}^{T}(\bar{X})+\boldsymbol{Z}(\bar{X})
$$

The first term models the trend (mean) of the output by a set of basis functions $\bar{f}_{K R G}(\bar{X})=$ $\left[f_{1}(\bar{X}), \ldots, f_{n}(\bar{X})\right]$ and associated regression coefficients $\bar{\beta}_{K R G}=\left[\beta_{1}, \ldots, \beta_{n}\right]$. The second term is interpolating the known residuals at the experimental design by a stationary zero mean Gaussian process fully described by its covariance (cov):

$$
\operatorname{cov}\left(\bar{X}, \bar{X}^{\prime}\right)=\sigma_{K R G}^{2} R\left(\bar{X}, \bar{X}^{\prime}, \bar{\theta}_{R}\right)
$$

Here $\sigma_{K R G}^{2}$ is the overall process variance (assumed constant) and $R$ models the correlation between $\boldsymbol{Z}(\bar{X})$ and $\boldsymbol{Z}\left(\bar{X}^{\prime}\right)$ by their inter-distance and a correlation function defined by the hyper parameters $\bar{\theta}_{R}$. Once a suitable basis of functions and a correlation model are chosen $\bar{\beta}_{K R G}$, $\sigma_{K R G}^{2}$, and $\bar{\theta}_{R}$ may be estimated by maximizing the likelihood of observing the output at the experimental design [34]. 
A priori it is known that the sensitivity between fatigue loads and the different wind climate parameters varies significantly. An anisotropic separable correlation formulation is therefore considered as outlined by Eq. (14).

$$
R\left(\bar{X}, \bar{X}^{\prime}, \bar{\theta}_{R}\right)=\prod_{i=1}^{D} R\left(X_{i}, X_{i}^{\prime}, \theta_{R, i}\right)
$$

A main challenge when calibrating an accurate Kriging model is to select an appropriate trend and correlation function. By a combinatorial approach similar to Morató et al. [9] we found that universal Kriging with a quadratic trend and the Matérn 3/2 correlation function yielded the best results overall.

\section{Method for Assessment of Surrogate Model Uncertainty}

The surrogate model uncertainties of PCE and Kriging are estimated according to EN 1990, Annex D [35]. This section briefly outlines the method followed by an in-depth description of the numerical integration scheme that is used to assess the site-specific fatigue loads.

\subsection{EN 1990 method}

The model uncertainty, $\boldsymbol{X}_{\text {proxy }}$, is estimated by rewriting it in terms of a unit mean lognormal error $\left(\boldsymbol{X}_{\text {proxy,EN }}\right)$ and a mean value correction factor to account for the model bias ( $\left.b_{\text {proxy }}\right)$, see Eq. (15).

$$
F_{\text {eq, direct }}=b_{\text {proxy }} \boldsymbol{X}_{\text {proxy }, E N} F_{\text {eq,proxy }}
$$

Given the available set of 99 statistically independent ${ }^{\mathrm{e}}$ joint wind climates the bias is estimated by a least squares approach as shown in Eq. (16).

$$
b_{\text {proxy }}=\frac{\sum_{i=1}^{99} F_{\text {eq, direct }, i} F_{\text {eq }, \text { proxy }, i}}{\sum_{i=1}^{99} F_{\text {eq,proxy }, i}^{2}}
$$

Next, the logarithm of the residuals at each site $\left(\delta_{E N, i}\right)$ is estimated by Eq. (17).

$$
\delta_{E N, i}=\ln \left(\frac{F_{\text {eq, direct }, i}}{b_{\text {proxy }} F_{\text {eq, direct }, i}}\right)
$$

The standard deviation of the residuals $\left(\sigma_{\delta, E N}\right)$ is then assessed by Eq. (18) where $\delta_{E N, \mu}$ is the mean value of all error realizations.

\footnotetext{
e The diversity of the included sites in terms of complexity and geographical spread validates the assumption of independence.
} 


$$
\sigma_{\delta, E N}=\sqrt{\frac{1}{99-1} \sum_{i=1}^{99}\left(\delta_{E N, i}-\delta_{E N, \mu}\right)^{2}}
$$

Finally, the coefficient of variation of the lognormal surrogate model uncertainty $\left(V_{\text {proxy }}\right)$ is calculated by Eq. (19).

$$
V_{\text {proxy }}=\sqrt{e^{\sigma_{\delta, E N}^{2}}-1}
$$

\subsection{Numerical fatigue load integration}

To evaluate the surrogate model uncertainty, it is necessary to assess $F_{e q}$ by direct simulation. This is not trivial and involves hundreds of thousands of aero-elastic simulations, hence, it is relevant to discuss the applied method to integrate the fatigue load in detail.

With flow inclination being fixed $\left(\varphi_{f i x}\right)$ the dimension of the integration problem in Eq. (7) is reduced as shown in Eq. (20). Here, the 12 discrete directions are directly introduced and $\bar{C}_{R}=$ $[U, \alpha, \rho]$ contain the remaining set of wind climate parameters of wind speed, wind shear and air density.

$$
\begin{aligned}
& F_{\text {eq }}\left(\varphi_{\text {fix }}\right) \\
& =(\frac{T_{\text {ref }}}{T_{\text {sim }}} \sum_{k=1}^{12} P_{\theta}\left(\theta_{k}\right) \underbrace{\int_{\sigma_{U}} \int_{\bar{C}_{R} \in \mathbb{R}^{3}} f_{\text {Site }}\left(\bar{C}_{R}, \sigma_{U} \mid \theta_{k}\right) \operatorname{DEL}\left(\bar{C}_{R}, \sigma_{U, T}, \varphi_{\text {fix }}\right)^{m} d \bar{C}_{R} d \sigma_{U}}_{F_{\text {eq,sect }, k}^{m}})^{\frac{1}{m}}
\end{aligned}
$$

To find an optimal numerical integration scheme it is sufficient to consider the sector wise fatigue loads $\left(F_{\text {eq,sect }, k}\right)$, which mathematically is the $\mathrm{m}^{\text {th }}$ order weighted Hölder mean of $D E L$ with respect to $f_{\text {Site }}$. In turn, $F_{\text {eq,sect, }, k}^{m}$ is the expected value of $D E L^{m}$ which may be estimated approximatively by Monte-Carlo ( $M C$ ) sampling, and thereby avoid the "curse of dimensionality" associated to traditional grid-based integration [6]. The $M C$-integration is outlined in Eq. (21), where $N$ is the number of samples and $h_{M C}$ models the probability of generating sample $i$ in terms of ambient turbulence.

$$
F_{e q, s e c t, k}^{m}\left(\varphi_{f i x}\right) \approx \frac{1}{N} \sum_{i=1}^{N} \frac{f_{\text {Site }}\left(\bar{C}_{R, i}, \sigma_{U, i} \mid \theta_{k}\right) \operatorname{DEL}\left(\bar{C}_{R, i}, \sigma_{U, T, i}, \varphi_{\text {fix }}\right)^{m}}{h_{M C}\left(\bar{C}_{R, i}, \sigma_{U, i}\right)}
$$


The convergence of Eq. (21) depends on the choice of the $M C$-sampling distribution. In principle, convergence is obtained more quickly if it resembles the product of $D E L^{m}$ and $f_{\text {Site }}$ to concentrate samples in the region which contributes most to the integral (i.e. importance sampling). This leads to the following key considerations for $h_{M C}$ :

1. Since $D E L$ s are raised to the power of $m$ it is important to sample high fatigue load events with a low probability of occurrence.

2. To capture the majority of the wind turbine's lifetime it is necessary to sample wind climate combinations with a high probability of occurrence.

The consequence of point one was clearly demonstrated by Graf et al. [6] who benchmarked $M C$-integration by sampling from the joint wind climate distribution (i.e. $h_{M C}=f_{\text {Site }}$ ) which lead to very slow convergence rates for components with high Wöhler exponents.

Optimizing $h_{M C}$ with respect to point one is sensor-specific but generally it requires unlikely wind climates to be sampled (e.g. very high turbulence or wind shear). By contrast, optimization of $h_{M C}$ with respect to point two is site-specific and requires likely wind climates to be sampled. To cover all 99 sites, and all considered sensors, a straight-forward compromise is to sample with equal density across the entire input domain defined in Section 4 (i.e. $h_{M C}$ uniformly distributed). As this sampling strategy is completely independent of $f_{\text {Site }}$ it also holds the advantage that the same $M C$-samples can be used across all sites, and thereby significantly reducing the required $D E L$ evaluations. It is noted that to reuse $D E L$ s across all sectors at a given site, ambient as well as wake-affected, the sampled turbulence has to be interpreted as total turbulence. To account for this, a Rosenblatt transformation is used to derive the total turbulence sample distribution given wind speed in wake-affected sectors (which is uniform for the defined input domain). The change-of-variable technique is then used to assess $h_{M C}$ in terms of ambient turbulence by applying the inverse of the transformation in Eq. (5) where $\theta$ and $U$ are fixed. A downside of this procedure is that all $M C$-samples with turbulence values less than $\sigma_{U, w a k e}$ become invalid in wake-affected sectors as they correspond to imaginary ambient turbulence. In turn, wake-affected sectors are effectively evaluated by fewer samples than ambient sectors, which is properly accounted for in all proceeding calculations.

Several techniques can be used to sample from $h_{M C}$ but in low dimensions (less than six) Morokoff and Catflisch [36] showed that quasi-random numbers from low-discrepancy sequences provide fast convergence. Instead of a convergence ratio $\propto N^{-0.5}$ for crude $M C$ integration, a Halton sequence obtains a convergence ratio $\propto N^{-\lambda}$ where $0.5 \leq \lambda \leq 1$ with $\lambda \rightarrow 1$ as dimensions reduce. The main question to answer is how many samples are required to accurately assess $F_{e q}$ in the current setup. Based on the results of Graf et al. [6] and a preliminary convergence study using a surrogate model to predict DELS it was found reasonable to evaluate the integral by 25,000 samples $^{\mathrm{f}}$. This resulted in an accuracy within approximately $1 \%$ of the converged value obtained at one million samples for all considered sensors and across all sites. Given that the surrogate model uncertainty is obtained by a relative

\footnotetext{
${ }^{\mathrm{f}}$ When a neighbouring turbine is $5 R D$ away this corresponds to an effective number of approximately 16,000 samples. For $3 R D$ it corresponds to approximately 12,500 samples.
} 
comparison of $F_{\text {eq,direct }}$ and $F_{\text {eq,proxy }}$, both estimated by the same set of $M C$-samples, the small error is assumed to be insignificant. Finally, it is noted that each sample in the $M C$ integration corresponds to one specific flow inclination so three databases of fatigue loads were simulated to cover all 99 sites. Using 100 seeds to estimate each $D E L$ this resulted in a total of 7.5 million $10 \mathrm{~min}$. simulations to accurately assess $F_{\text {eq, direct }}$.

\section{Quantification of the Surrogate Model Uncertainty}

This section presents a quantification of the surrogate model uncertainty. First, an example is shown step-by-step for the blade root flap-wise bending moment to clearly outline the procedure. Thereafter, the surrogate model uncertainty is quantified for all considered sensors. It is noted that only the worst-case uncertainty and bias across the sensors will be highlighted as it is impractical to differentiate the surrogate model uncertainty for each sensor ${ }^{\mathrm{g}}$.

\subsection{Blade root bending moment example}

Using the full experimental design, a Kriging and a PCE model is trained to capture fatigue loads of the blades. Both surrogate models are then used to estimate the site-specific integrated fatigue loads at all 99 sites by Eq. (20), which is compared to direct simulation as illustrated in Figure 4.
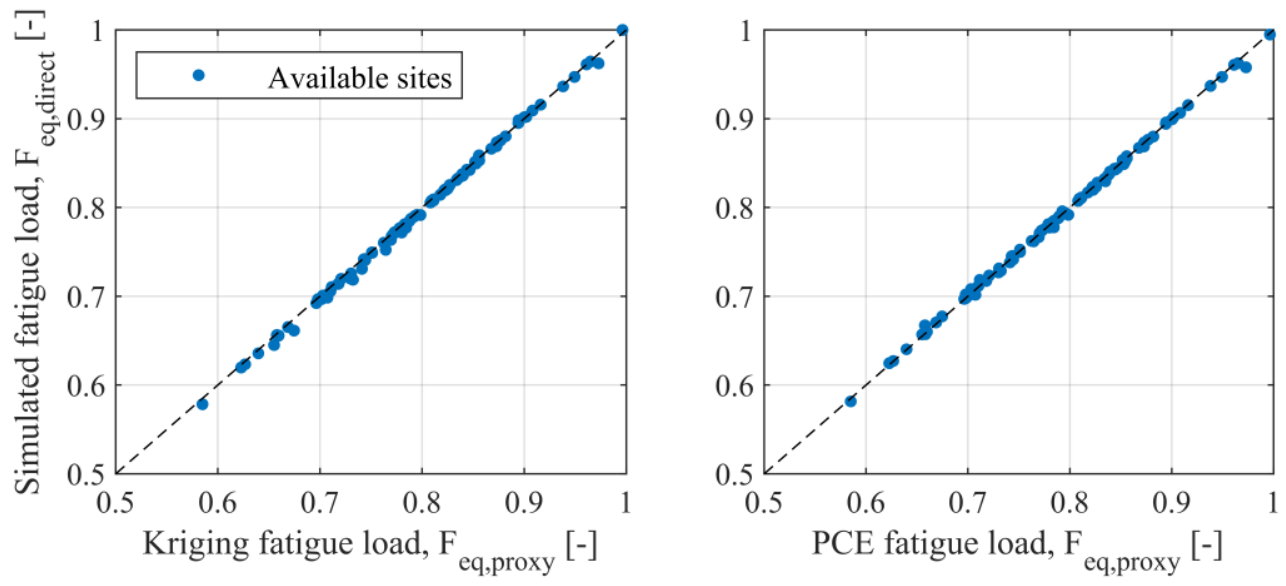

Figure 4: Normalized site-specific fatigue loads on the blades predicted by Kriging and PCE and compared to direct simulation. The dashed lines indicate the perfect model where $F_{\text {eq,proxy }}=F_{\text {eq,direct }}$

Based on this comparison the surrogate model uncertainty is estimated by Eqs. (16) and (19). The results are presented in Table 5. Given the typical scale of the other relevant uncertainties on fatigue strength and load effects listed in Table 4, the surrogate model uncertainties are insignificant in this present case and may be neglected.

\footnotetext{
$\mathrm{g}$ This representation of the surrogate model uncertainty is in line with the model uncertainty for wind load effects being identical across all considered sensors.
} 
Table 5: Surrogate model uncertainty for blade flap-wise bending.

\begin{tabular}{|l|l|l|}
\hline Surrogate model & Bias [-] & Coefficient of variation [-] \\
\hline PCE & 1.001 & 0.004 \\
\hline Kriging & 1.004 & 0.004 \\
\hline
\end{tabular}

Next, we investigate how the results change with the simulation effort used to train the surrogate models. This is illustrated in Figure 5 where the coefficient of variation is plotted as function of the number of samples in the experimental design and how many seeds that are used to evaluate each corresponding $D E L$. Similarly, the model bias is shown as function of simulation effort in Figure 6. The number of samples starts at 100 as both surrogate models exhibit considerable inaccuracy for smaller experimental designs. The apparent noise in the PCE results is explained by the adaptive scheme used to select the optimal polynomial degree which may change for each combination of samples/seeds in order to minimize $\epsilon_{L O O}$.
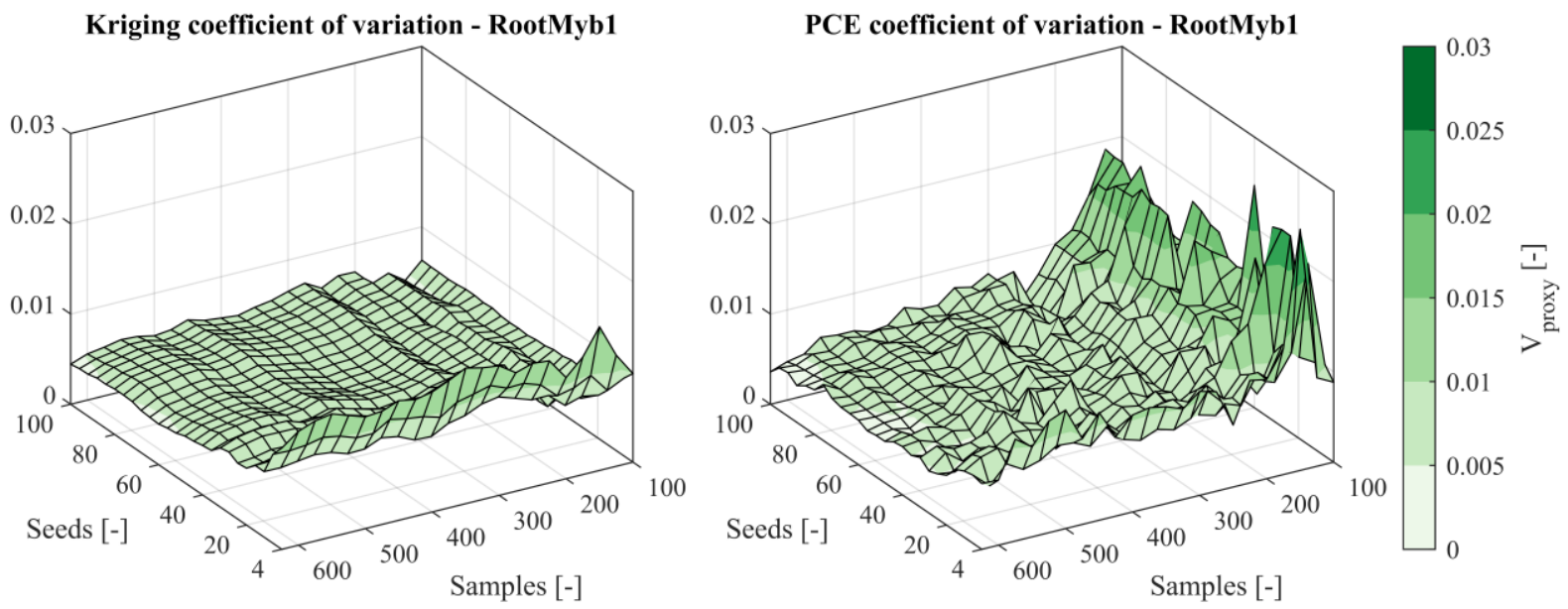

Figure 5: Coefficient of variation of the surrogate model uncertainty for the blades using Kriging and PCE shown as function of the number of samples used to train the models and the number of seeds used to evaluate the DEL at each sample. 

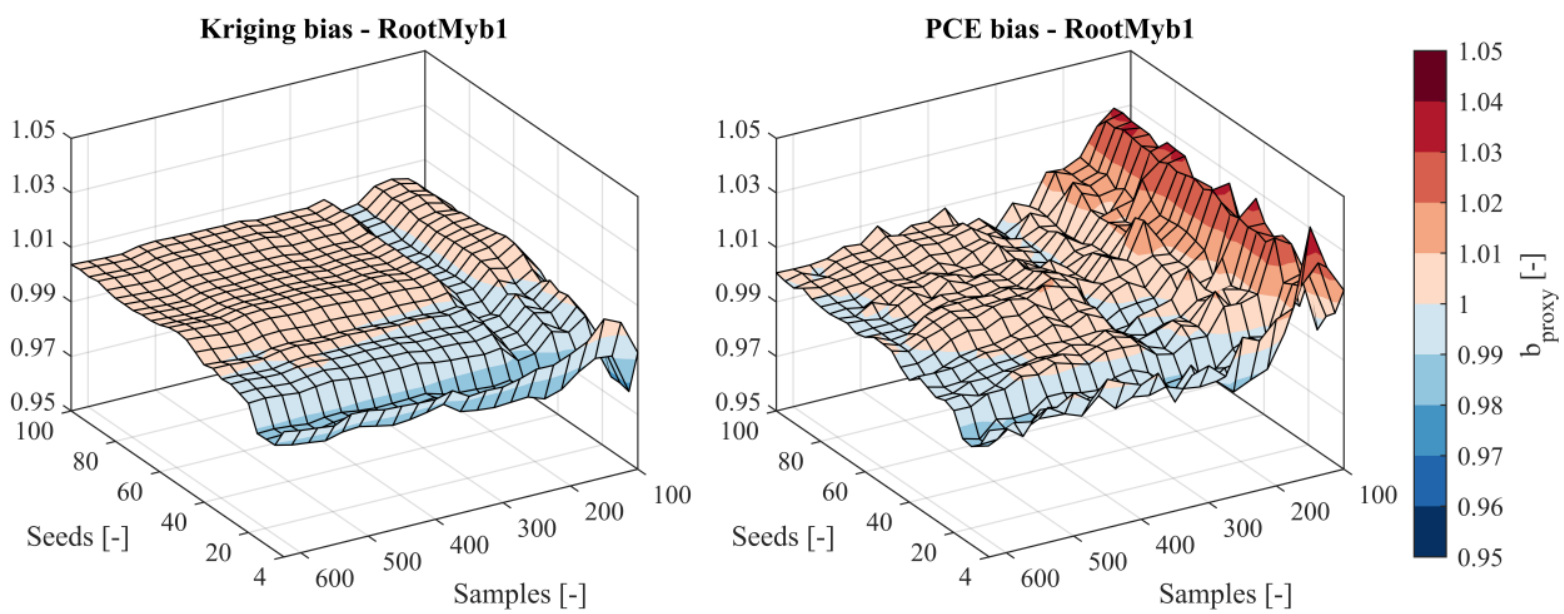

Figure 6: Surrogate model bias for the blades using Kriging and PCE shown as function of the number of samples used to train the models and the number of seeds used to evaluate the DEL at each sample. The apparent "valley" in the Kriging results at approximately 200 samples is due to the oscillating convergence behaviour of the bias across samples. This is clearly seen when less than 100 samples are considered, but to keep the figures consistent and clean this is not included here.

\subsection{Surrogate model uncertainty across all sensors}

Following the procedure described for the blade flap-wise bending moment the surrogate model uncertainties have been evaluated for all sensors in Table 3. The maximum coefficient of variation across the sensors is plotted in Figure 7, demonstrating how relatively few samples and seeds are required for both surrogate models to converge at approximately $\sim 0.5 \%$.
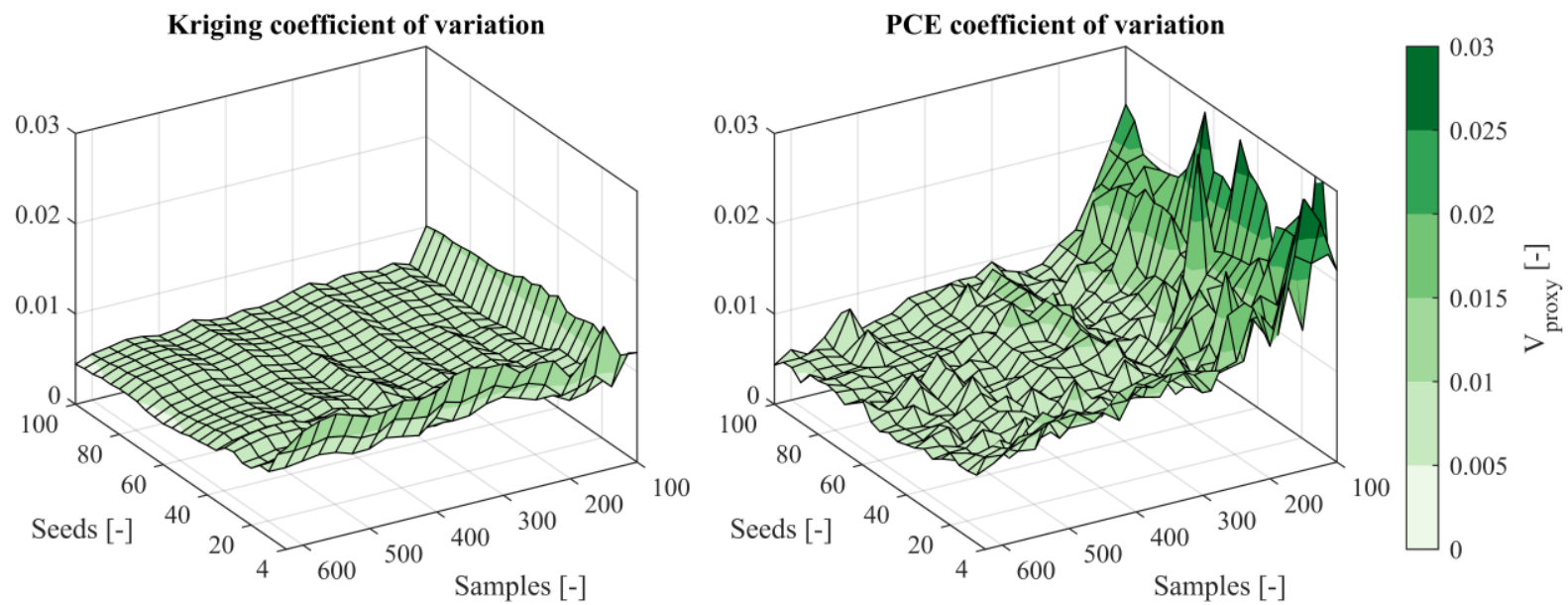

Figure 7: Surrogate model uncertainty coefficient of variations across all considered sensors shown as function of the number of samples used to train the models and the number of seeds used to evaluate the DEL at each sample.

To analyse the surrogate model bias it is necessary to consider the minimum and maximum values across all sensors as illustrated in Figure 8 and Figure 9, respectively. This shows that for sparse designs (low number of samples) PCE tends to be non-conservative while Kriging seems to be almost exclusively conservative across all sensors. 

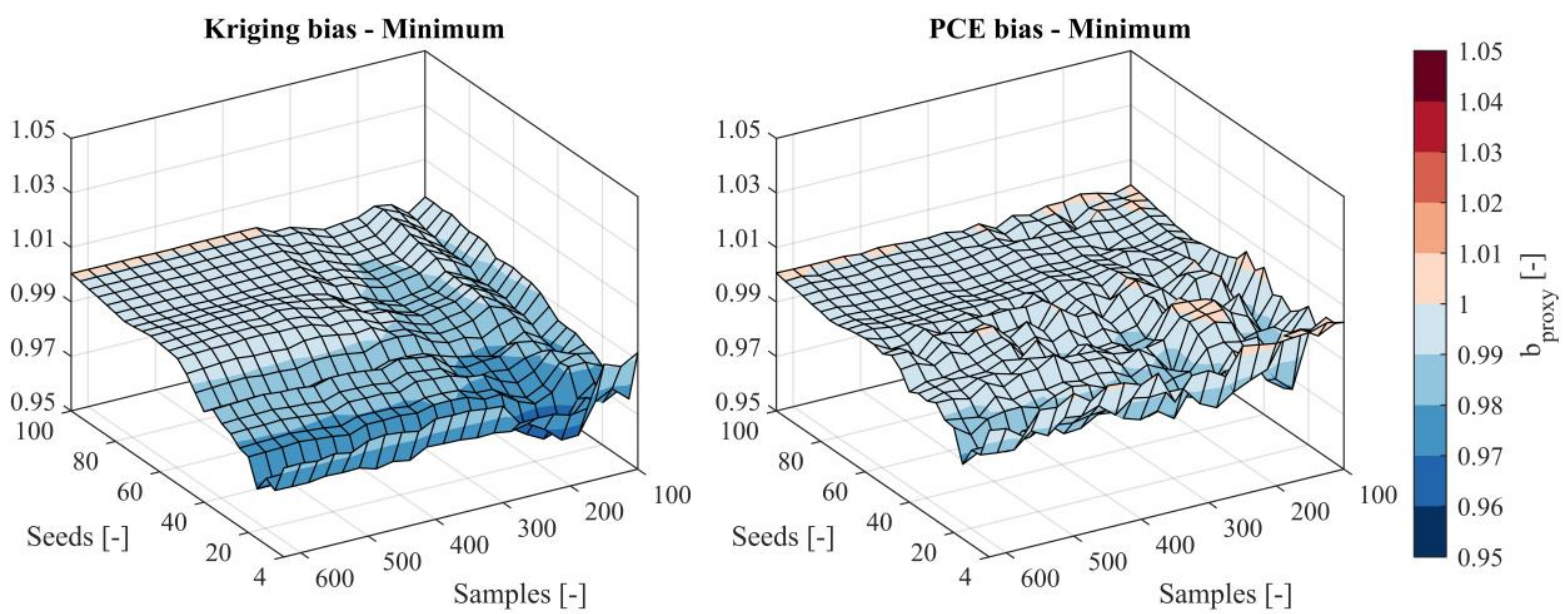

Figure 8: Minimum surrogate model bias across all considered sensors shown as function of the number of samples used to train the models and the number of seeds used to evaluate the DEL at each sample.
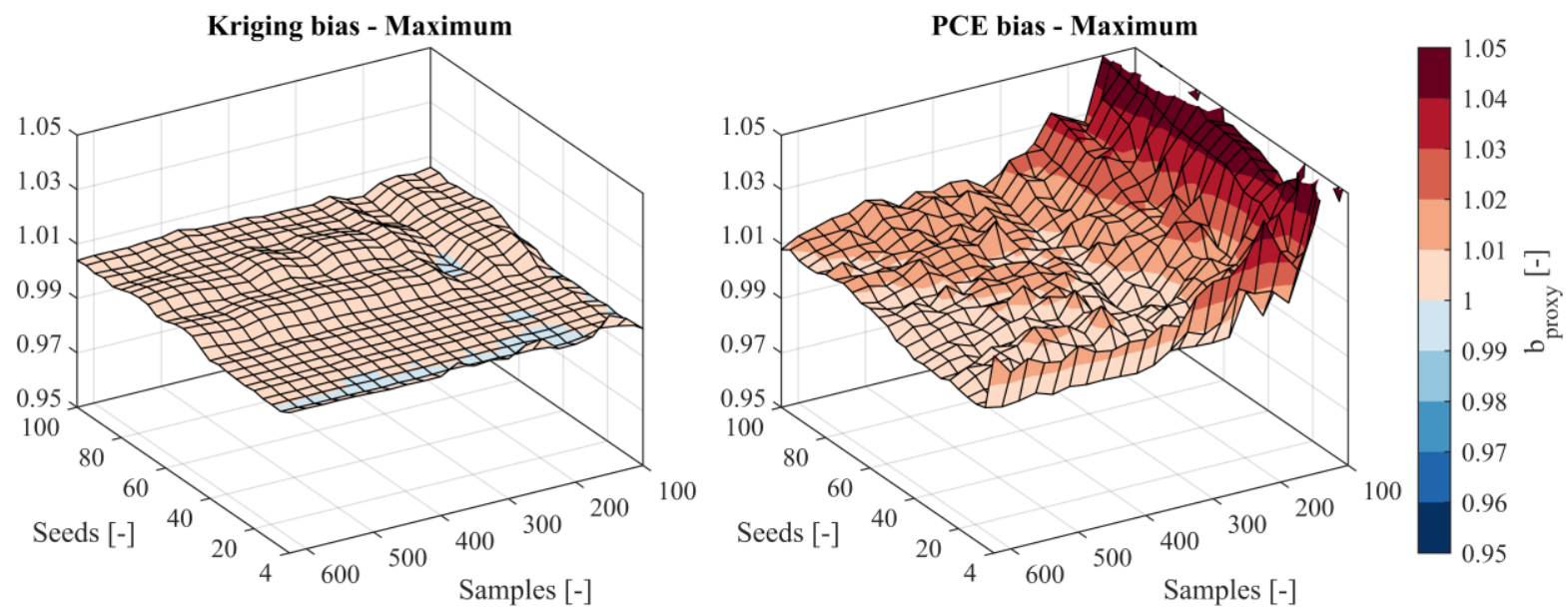

Figure 9: Maximum surrogate model bias across all considered sensors shown as function of the number of samples used to train the models and the number of seeds used to evaluate the DEL at each sample. The PCE model bias at 100150 samples is missing due to a sharp increase to 1.10 which would otherwise distort the figure.

\section{Sensitivity Analysis and Recommendations}

In this section a sensitivity analysis is conducted to assess how the surrogate model uncertainty affects the reliability level of the considered components. This is used to define three levels of accuracy followed by a set of recommendations by the authors on training PCE and Kriging for fatigue reliability analysis of wind turbines.

\subsection{Reliability sensitivity analysis}

To estimate the reliability sensitivity towards the surrogate model uncertainty a reference design $\left(z_{r e f}\right)$ is established by assuming that each component is designed to the limit using direct aero- 
elastic simulation (i.e. using the LSE in Eq. (8)). This implies that the reliability index at the last year of service is exactly the target of $\Delta \beta_{t}\left(z_{\text {ref }}, T_{\text {life }}\right)=3.3$ [1], where $T_{\text {life }}$ is the assumed turbine lifetime of 20 years. The annual probability of failure is approximated as $\Delta P_{f, 20} \cong$ $P_{f, 20}-P_{f, 19}$ where $P_{f, 20}$ and $P_{f, 19}$ represent the accumulated failure probabilities at years 19 and 20, respectively, and all the uncertainties are modelled according to Table 4. Using the reference design for each component the reliability index at the final year of service $(\Delta \beta)$ is calculated by the LSE in Eq. (10), where $b_{\text {proxy }}$ and $V_{\text {proxy }}$ are varied individually. Subsequently, the sensitivity towards the surrogate model uncertainty is quantified as the ratio $\Delta \beta / \Delta \beta_{t}$. In Figure 10 (left) this is shown for a representative range of $V_{\text {proxy }}$, demonstrating that the relative change of the reliability index is less than $0.5 \%$. Figure 10 (right) shows the results of varying $b_{\text {proxy }}$, which reveals the important relationship that $\Delta \beta / \Delta \beta_{t} \cong b_{\text {proxy }}$ for all components considered.
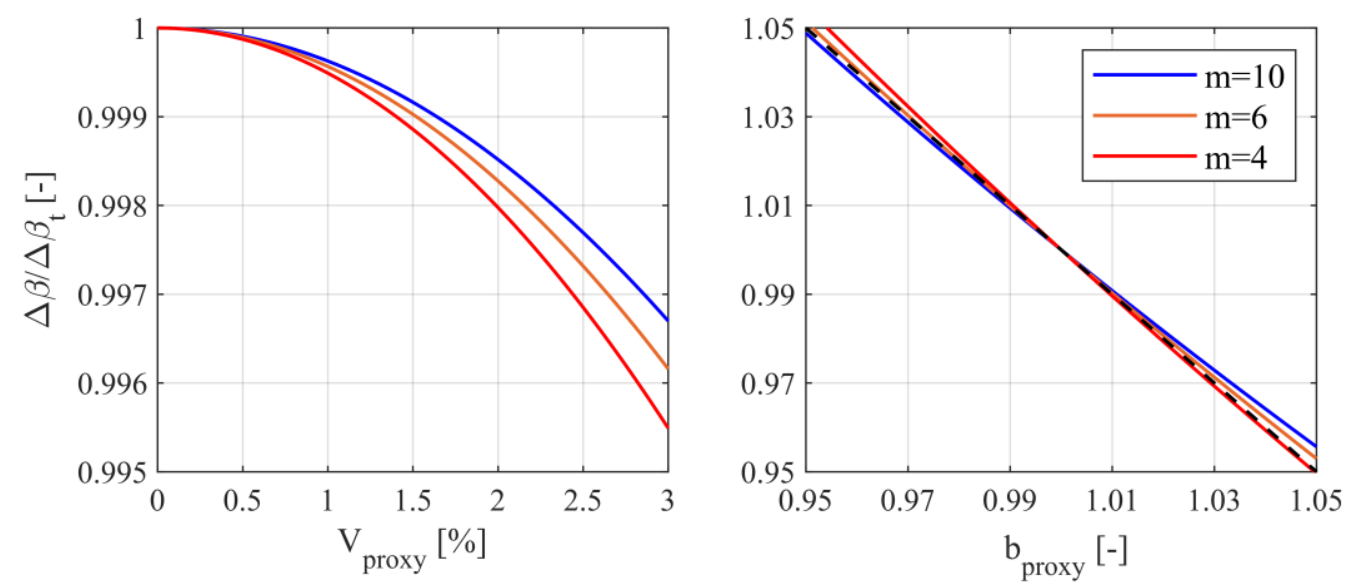

Figure 10: Reliability index sensitivity towards the surrogate model uncertainty coefficient of variation. The dashed line on the plot to the right indicates $\Delta \beta / \Delta \beta_{t}=b_{\text {proxy }}$.

\subsection{Surrogate model accuracy}

Typically, the target reliability index is specified with one decimal in standards and codes, thereby suggesting that less than $1 \%$ change of the reliability index at the limit is negligible. $V_{\text {proxy }}$ may, therefore, be ignored in reliability analysis of wind turbines for both Kriging and PCE when at least 100 samples are considered in the experimental design. The change in reliability is approximately proportional to $b_{\text {proxy }}$, thereby making the bias significant when it is outside the range of 0.99 to 1.01 . This is also clearly shown in Table 6 which is used to define three accuracy classes in the following.

Table 6: Absolute reliability for varying surrogate model bias.

\begin{tabular}{|c|l|l|l|l|l|l|l|l|l|l|l|}
\hline$b_{\text {proxy }}$ & 0.95 & 0.96 & 0.97 & 0.98 & 0.99 & 1.00 & 1.01 & 1.02 & 1.03 & 1.04 & 1.05 \\
\hline$\Delta \beta$ & 3.1 & 3.2 & 3.2 & 3.2 & 3.3 & 3.3 & 3.3 & 3.4 & 3.4 & 3.4 & 3.5 \\
\hline
\end{tabular}


High accuracy is obtained when $0.99 \leq b_{\text {proxy }} \leq 1.01$ where the estimated reliability index is unchanged on the first decimal. Medium accuracy is obtained when $0.96 \leq b_{\text {proxy }} \leq 1.04$ leading to a slight change of \pm 0.1 for the estimated reliability index. If $0.96>$ $b_{\text {proxy }}$ or $b_{\text {proxy }}>1.04$ low accuracy is obtained which potentially changes the estimated reliability index drastically by more than \pm 0.2 . The accuracy classes are pictured for each surrogate model in Figure 11.
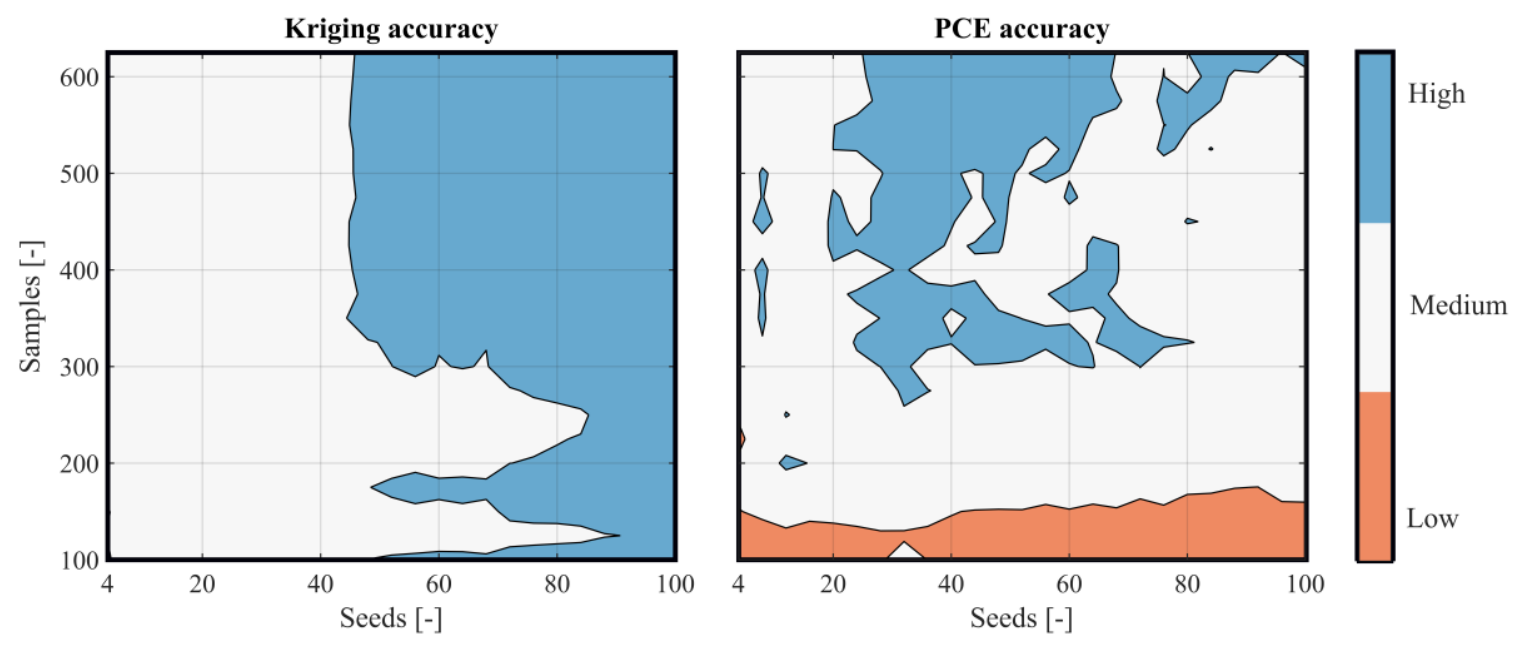

Figure 11: Surrogate model bias divided into three accuracy classes. Notice the clear pattern of increasing accuracy with the number of seeds for Kriging and the increased accuracy with the number of samples for PCE.

For Kriging the accuracy depends highly on the number of seeds that are used. The upper right corner confirms a converged plateau of high accuracy which requires an experimental design with more than 350 samples using at least 50 seeds to assess each $D E L$. If a medium accuracy is acceptable it is only required to use more than 100 samples and at least 4 seeds.

For PCE the accuracy depends on the density of the experimental design. A high accuracy may be obtained, but even when the full design is used the accuracy is not fully consistent with an increase in seeds. This indicates that the PCE coefficients may not have converged fully in the investigated range of simulation effort. If more than 175 samples are considered the PCE model reaches a medium accuracy, and low accuracy is obtained for experimental designs with less than $~ 175$ samples.

\subsection{Recommendations}

The results of the optimization analysis are summarized in Tables 7 and 8 as a set of recommendations to train Kriging and PCE for fatigue reliability analysis of onshore wind turbines. Since only a subset of load-bearing components is considered the recommendations are deliberately conservative compared to the results shown in Figure 11.

It is important to recognise that the recommendations are tied to the current setup of using the $5 \mathrm{MW}$ reference turbine designed by NREL together with the experimental design described in Section 4. The minimum samples and seeds are also dependent on the size of the input domain, which directly relates to the sample density. If a similar experimental design is used together 
with a smaller or larger input domain emphasis should be on the recommended maximum "leave one out" error. Since the proposed experimental design is uniformly distributed without any emphasis on the turbine specific $D E L$ response it is expected that the recommended maximum "leave one out" error can be used as tentative guidance for other turbines of similar architecture.

Table 7: Kriging recommendations

\begin{tabular}{|l|l|l|l|l|}
\hline Accuracy & $\begin{array}{l}\text { Minimum samples } \\
\text { in DoE [-] }\end{array}$ & $\begin{array}{l}\text { Minimum seeds to } \\
\text { assess DEL [-] }\end{array}$ & $\begin{array}{l}\text { Total amount of 10 } \\
\text { min. simulations [-] }\end{array}$ & $\begin{array}{l}\text { Maximum } \\
\epsilon_{\text {Loo }}[\%]\end{array}$ \\
\hline High & 400 & 75 & 30,000 & 0.60 \\
\hline Medium & 100 & 10 & 1,000 & 2.00 \\
\hline Low & 100 & 4 & 400 & 3.25 \\
\hline
\end{tabular}

Table 8: PCE recommendations

\begin{tabular}{|l|l|l|l|l|}
\hline Accuracy & $\begin{array}{l}\text { Minimum samples } \\
\text { in DoE [-] }\end{array}$ & $\begin{array}{l}\text { Minimum seeds to } \\
\text { assess DEL [-] }\end{array}$ & $\begin{array}{l}\text { Total amount of 10 } \\
\text { min. simulations [-] }\end{array}$ & $\begin{array}{l}\text { Maximum } \\
\epsilon_{\text {Loo }}[\%]\end{array}$ \\
\hline High & $>625$ & 50 & $>31,250$ & 0.35 \\
\hline Medium & 200 & 10 & 2,000 & 1.35 \\
\hline Low & 100 & 4 & 400 & 2.20 \\
\hline
\end{tabular}

\section{Comparison and Discussion of PCE and Kriging}

Overall, Kriging obtains a higher accuracy than PCE per aero-elastic simulation, but it also requires more computations to predict new samples [7]. However, as stressed previously $h_{M C}$ is completely independent of the joint wind climate distribution and the load response function in the proposed numerical integration. A direct advantage of this is that the $D E L$ of each $M C$ sample has to be evaluated only once per fixed flow inclination to cover all 99 sites. In practice, this strategy therefore neglects the difference in computational time between PCE and Kriging, when compared to the simulation time invested into training the models.

To increase the accuracy of PCE it requires more samples while Kriging requires more seeds. This observation is in line with the underlying fundamentals of the surrogate techniques of regression and interpolation, respectively. If few seeds are used to evaluate the $D E L$ s it can be interpreted as noise which Kriging is forced to capture when interpolating the residuals. By contrast, PCE levels out the noise in a mean sense, but a relatively large number of samples are needed to reliably fit the coefficients of the expansion. Although not shown in this paper, this encouraged an investigation of using Polynomial Chaos Kriging [37] (PCK) as implemented in UQLab [38]. This method combines the two surrogate models by using the PCE as basis functions (trend) for the universal Kriging model. In the best-case scenario this would lead to a 
superior surrogate model which is accurate for small experimental designs (as Kriging) using just a few seeds to estimate DELs (as PCE). However, the results of using PCK mostly resemble those obtained using PCE, and it is therefore not preferred over traditional universal Kriging. The reason is probably that the residuals used to estimate $\sigma_{K R G}^{2}$ and $\bar{\theta}_{R}$ in PCK appear without any significant correlation, and to a large degree they just represent the noise from using few seeds. In turn, the Kriging interpolation only influences predictions at the very vicinity of the experimental design, which was also observed by Dimitrov et al. [7].

Another possibility to increase the accuracy of Kriging is to introduce a so-called "nugget". The nugget models a set of values that are added to the diagonal of $R\left(\bar{X}, \bar{X}^{\prime}, \bar{\theta}_{R}\right)$, thereby allowing a non-zero uncertainty bound around the experimental design. A well-optimized nugget could therefore potentially make the Kriging model more robust against the apparent noise that is introduced when a small number of seeds is considered [39]. However, this approach was not pursued further in this work and is as such open for continued research.

\section{Summary and Conclusions}

The model uncertainty related to approximating lifetime fatigue loads by Kriging and PCE has been quantified using wind data from 99 international sites and based on aero-elastic simulations of NREL's 5MW reference turbine. The main components of the turbine were considered, namely the blades, the drivetrain, the yaw bearing and the tower.

An experimental design to train the surrogate models in terms of wind speed, turbulence, wind shear exponent, air density and flow inclination was defined by all the available data. Using up to 625 samples, and up to 100 seeds to evaluate fatigue loads at each sample, both surrogate models were calibrated in the software UQLab. For Kriging, a combinatorial approach was used to conclude that universal Kriging with a second order trend, in conjunction with the Matern $3 / 2$ correlation model, is optimal to predict fatigue loads.

The model uncertainty of both surrogate techniques across all sensors was estimated by the recommended approach in Eurocode 1990, Annex D. This revealed that the surrogate model uncertainty coefficient of variation is less than $2.5 \%$. Based on a sensitivity study it was shown how the model uncertainty coefficient of variation changes the structural reliability index less than $0.5 \%$, which is practically negligible compared to the target reliability index of 3.3. In contrast, the model bias varied significantly for the two surrogate models between 0.95 to 1.05 , and the sensitivity study demonstrated that the relative change in reliability index is approximately equal to the model bias. It is, therefore, critical to avoid significantly biased surrogate models in site-specific fatigue reliability assessments of wind turbines.

Three accuracy classes were introduced based on the surrogate model bias, namely low (bias larger than $4 \%$ ), medium (bias less than $4 \%$ but more than $1 \%$ ), and high (bias less than $1 \%$ ). Compared to direct simulation, a surrogate model with a high accuracy estimated reliability indices within \pm 0.05 , for medium accuracy the error is within \pm 0.15 , and for low accuracy the error is $\geq 0.15$.

It was documented that Kriging yields a high accuracy for the considered 5MW reference turbine when more than 30,000 aero-elastic simulations are invested to train the surrogate model. In comparison, PCE did not consistently obtain a high accuracy in the investigated range 
of 625 samples and 100 seeds, but by using more than 200 samples and 10 seeds PCE achieved a medium accuracy. Altogether, this makes Kriging the preferred method for fatigue reliability analysis of onshore wind turbines when using the methods proposed by this paper.

\section{Acknowledgements}

The authors wish to thank the data providers: KNMI, ICDC, CliSAP/KlimaCampus, University of Hamburg, DTU, Vattenfall and VENTUS INGENIERÍA. The work presented in this paper is part of the $\mathrm{PhD}$ project "From wind climate to wind turbine loads - efficient and accurate decision support and reliability analysis" co-funded by EMD International A/S, Aalborg University and the Innovationfund Denmark case number 5189-00022B. Their financial support is greatly appreciated.

\section{References}

[1] IEC., International Standard IEC 61400-1 ed. 4, "Wind Turbines - Part 1 Design Requirements"., (2019).

[2] ISO., International Standard ISO 2394:2015, “General principles on reliability for structures," (2015).

[3] R.M.M. Slot, L. Svenningsen, J.D. Sørensen, M.L. Thøgersen, Importance of Shear in Site Assessment of Wind Turbine Fatigue Loads, J. Sol. Energy Eng. 140 (2018) 041012. https://doi.org/10.1115/1.4039748.

[4] H. Stensgaard Toft, L. Svenningsen, W. Moser, J. Dalsgaard Sørensen, M. Lybech Thøgersen, Wind Climate Parameters for Wind Turbine Fatigue Load Assessment, J. Sol. Energy Eng. 138 (2016). https://doi.org/10.1115/1.4033111.

[5] IEC., International Standard IEC 61400-1 ed. 3, "Wind Turbines - Part 1 Design Requirements"., (2010).

[6] P.A. Graf, G. Stewart, M. Lackner, K. Dykes, P. Veers, High-throughput computation and the applicability of Monte Carlo integration in fatigue load estimation of floating offshore wind turbines, Wind Energy. $19 \quad$ (2016) 861-872. https://doi.org/110.1002/we.1870.

[7] N. Dimitrov, M. Kelly, A. Vignaroli, J. Berg, From wind to loads: wind turbine sitespecific load estimation using databases with high-fidelity load simulations, Wind Energy Sci. 3 (2018) 767-790. https://doi.org/10.5194/wes-2018-18.

[8] H.S. Toft, L. Svenningsen, J.D. Sørensen, W. Moser, M.L. Thøgersen, Uncertainty in wind climate parameters and their influence on wind turbine fatigue loads, Renew. Energy. 90 (2016) 352-361. https://doi.org/10.1016/j.renene.2016.01.010.

[9] A. Morató, S. Sriramula, N. Krishnan, Kriging models for aero-elastic simulations and reliability analysis of offshore wind turbine support structures, Ships Offshore Struct. 0 (2018) 1-14. https://doi.org/10.1080/17445302.2018.1522738.

[10] R. Teixeira, A. O’Connor, M. Nogal, N. Krishnan, J. Nichols, Analysis of the design of 
experiments of offshore wind turbine fatigue reliability design with Kriging surfaces, Procedia Struct. Integr. 5 (2017) 951-958. https://doi.org/10.1016/j.prostr.2017.07.132.

[11] J.P. Murcia, P. Réthoré, N. Dimitrov, A. Natarajan, J.D. Sørensen, P. Graf, T. Kim, Uncertainty propagation through an aeroelastic wind turbine model using polynomial $\begin{array}{lllll}\text { surrogates, } & \text { Renew. } & \text { Energy. } & 119 & \text { (2017) 910-922. }\end{array}$ https://doi.org/10.1016/j.renene.2017.07.070.

[12] W. Hu, K.K. Choi, H. Cho, Reliability-based design optimization of wind turbine blades for fatigue life under dynamic wind load uncertainty, Struct. Multidiscip. Optim. 54 (2016) 953-970. https://doi.org/10.1007/s00158-016-1462-x.

[13] N. Dimitrov, A. Natarajan, M. Kelly, Model of wind shear conditional on turbulence and its impact on wind turbine loads, Wind Energy. 18 (2015) 1917-1931. https://doi.org/10.1002/we.1797.

[14] M. Shoaib, I. Siddiqui, S. Rehman, S. Khan, L.M. Alhems, Assessment of wind energy potential using wind energy conversion system, J. Clean. Prod. 216 (2019) 346-360. https://doi.org/10.1016/j.jclepro.2019.01.128.

[15] L. Svenningsen, R.M.M. Slot, M.L. Thøgersen, A novel method to quantify atmospheric stability, J. Phys. Conf. Ser. 1102 (2018). https://doi.org/10.1088/17426596/1102/1/012009.

[16] M. Kelly, G. Larsen, N.K. Dimitrov, A. Natarajan, Probabilistic Meteorological Characterization for Turbine Loads, J. Phys. Conf. Ser. 524 (2014) 012076. https://doi.org/10.1088/1742-6596/524/1/012076.

[17] M.A. Miner, Cumulative damage in fatigue, J. Appl. Mech. 12 (1945) 159-164.

[18] J.M. Jonkman, S. Butterfield, W. Musial, G. Scott, Definition of a 5-MW reference wind turbine for offshore system development, National Renewable Energy Laboratory, 2009.

[19] E.J. Novaes Menezes, A.M. Araújo, N.S. Bouchonneau da Silva, A review on wind turbine control and its associated methods, J. Clean. Prod. 174 (2018) 945-953. https://doi.org/10.1016/j.jclepro.2017.10.297.

[20] J. Jonkman, FAST An aeroelastic computer-aided engineering (CAE) tool for horizontal axis wind turbines, (2015). https://nwtc.nrel.gov/FAST [Accessed 2019-06-18] (accessed April 3, 2017).

[21] N.K. B. Jonkman, TurbSim A stochastic, full-field, turbulence simulator primarialy for use with InflowWind/AeroDyn-based simulation tools, (2016). https://nwtc.nrel.gov/TurbSim (accessed April 3, 2017).

[22] Standard, IEC 61400-1 ed. 3, 2005, International Electrotechnical Commission, Wind turbines, Part 1: Design requirements, Edition 3 (2005) incl. Amendment 1 (2010)., (2010).

[23] ASTM, ASTM No. E1049-85, "Standard Practice for Cycle Counting in Fatigue Analysis"., (2011).

[24] J.D. Sørensen, S. Frandsen, N.J. Tarp-Johansen, Effective turbulence models and fatigue reliability in wind farms, Probabilistic Eng. Mech. 23 (2008) 531-538. 
https://doi.org/10.1016/j.probengmech.2008.01.009.

[25] 61400-1 IEC, Safety Factors - IEC 61400-1 ed. 4 - background document, (2014).

[26] S. Marelli, B. Sudret, UQLab: A Framework for Uncertainty Quantification in Matlab, in: Vulnerability, Uncertainty, Risk, American Society of Civil Engineers, Reston, VA, 2014: pp. 2554-2563. https://doi.org/10.1061/9780784413609.257.

[27] T.J. Santner, B.J. Williams, W.I. Notz, The Design and Analysis of Computer Experiments, Springer New York, New York, NY, 2018. https://doi.org/10.1007/978-14939-8847-1.

[28] B. Sudret, Polynomial chaos expansions and stochastic finite element methods, in: K.K. Phoon, J. Ching (Eds.), Risk Reliab. Geotech. Eng., Taylor and Francis, 2015: pp. 265300.

[29] L. Kocis, W.J. Whiten, Computational investigations of low-discrepancy sequences, ACM Trans. Math. Softw. 23 (1997) 266-294. https://doi.org/10.1145/264029.264064.

[30] D. Xiu, G.E. Karniadakis, The Wiener--Askey Polynomial Chaos for Stochastic Differential Equations, SIAM J. Sci. Comput. 24 (2002) 619-644. https://doi.org/10.1137/S1064827501387826.

[31] M. Rosenblatt, Remarks on a Multivariate Transformation, Ann. Math. Stat. 23 (1952) 470-472.

[32] G. Blatman, B. Sudret, Adaptive sparse polynomial chaos expansion based on least angle regression, J. Comput. Phys. 230 (2011) 2345-2367. https://doi.org/10.1016/j.jcp.2010.12.021.

[33] S. Marelli, B. Sudret, UQLab user manual - Polynomial Chaos Expansions, Report UQLab-V1.0-105, ETH Zurich, 2017.

[34] C. Lataniotis, S. Marelli, B. Sudret, UQLab user manual - Kriging (Gaussian Process Modelling), Report UQLab-V1.0-105, ETH Zurich, 2017.

[35] CEN/TC, EN 1990-2002, Eurocode - Basis of structural design, (2002).

[36] W.J. Morokoff, R.E. Caflisch, Quasi-Monte Carlo Integration, J. Comput. Phys. (1995) $218-230$.

[37] R. Schöbi, B. Sudret, J. Wiart, Polynomial-chaos-based Kriging, Int. J. Uncertain. Quantif. 5 (2015) 171-193.

[38] S. Marelli, B. Sudret, UQLab user manual - PC-Kriging, Report UQLab-V1.0-105, ETH Zurich, 2017.

[39] I. Andrianakis, P.G. Challenor, The effect of the nugget on Gaussian process emulators of computer models, Comput. Stat. Data Anal. 56 (2012) 4215-4228. https://doi.org/10.1016/j.csda.2012.04.020. 